\title{
Observability-based Optimization for Flow Sensing and Control of an Underwater Vehicle in a Uniform Flowfield
}

\author{
Levi DeVries ${ }^{1}$ and Derek A. Paley ${ }^{2}$
}

\begin{abstract}
This paper describes how an underwater vehicle can control its motion by sensing the surrounding flowfield and using the sensor measurements in a dynamic feedback controller. Limitations in existing sensing modalities for flowfield estimation are mitigated by using a fish-inspired distributed sensor array and a nonlinear observer. Estimation performance is further increased by optimizing sensor placement on the vehicle body. We optimize sensor placement along a streamlined body using measures of flowfield observability, namely the empirical observability gramian. Velocity potentials model the flow around the vehicle and a recursive Bayesian filter estimates the flow from noisy velocity measurements. To orient the body into the oncoming flow (a fish-inspired behavior known as rheotaxis) we implement a dynamic, linear controller that uses the estimated angle of attack. Numerical simulations illustrate the theoretical results.
\end{abstract}

\section{INTRODUCTION}

The integration of bio-inspired sensing modalities with unmanned robotic systems has the potential to advance autonomous operations by enabling vehicles to better characterize the complex environments in which they operate. As new sensing technologies are developed and improved, [1],[2],[3],[4],[5] the performance of a distributed sensor array can be enhanced by optimizing the placement of the individual sensing nodes with respect to a desired objective [6],[7],[8],[9]. This paper presents an observability-based framework for evaluating fish-inspired sensor placement strategies for flowfield estimation, and demonstrates the use of the resulting sensor design in a closed-loop control system for an underwater vehicle. Specifically, we model the flow around a fish-shaped streamlined body outfitted with flow sensors and seek to optimize sensor placement configurations for estimating the surrounding flowfield. Estimation of the surrounding flowfield is an essential part of the design of a control system that stabilizes the upstream orientation of the body with respect to the flow, a fish-inspired behavior known as rheotaxis [10].

We model the potential flow around a streamlined body using conformal mapping [11]. This technique accurately models the flow at small angles of attack where flow separation is minimal. In light of related work [1] in which hair-sensor arrays are being developed to encode flow speed

*This work is supported by the Office of Naval Research under Grant No. N00014-12-1-0149.

${ }^{1}$ Levi DeVries is a Graduate Research Assistant in the Department of Aerospace Engineering at the University of Maryland, College Park, MD, 20742, USA Iddevrie@umd.edu

2 Derek A. Paley is an Associate Professor in the Department of Aerospace Engineering and the Institute for Systems Research at the University of Maryland, College Park, MD, 20742, USA dpaley@umd.edu and direction, analogous to the fish lateral line system [3],[10],[12], this paper considers multiple flow sensors that collect noisy measurements of the local flow velocity a finite distance from the body [1]. We optimize sensor placement using measures of flowfield observability as a scoring metric, assuming sensor hairs protrude sufficiently far from the vehicle such that boundary layer effects can be ignored. In the region near the vehicle but outside the boundary layer, the flow direction is predominantly tangent to the surface, which implies that a point measurement of flow velocity is a suitable representation of the hair sensor capability.

Candidate sensor placement configurations are evaluated using optimality measures based on the empirical observability gramian, also known as the empirical observability covariance matrix [6]. We show that for an analytic flowfield the empirical observability gramian is equal to the Fisher information matrix [13], scaled by the sensor noise covariance. By optimizing the configuration of the sensor array with respect to flowfield observability, estimation of the local flow around the vehicle is improved. This paper presents a recursive Bayesian filter for estimating the flowfield parameters from noisy measurements collected by the optimized sensor array. The estimated flowfield parameters are implemented in a dynamic feedback control algorithm to steer the body to the desired angle of attack with respect to the flow (which is zero for rheotaxis).

The contributions of this paper are (1) the application of an observability-based metric to the problem of evaluating and optimizing distributed sensor placement for flowfield estimation; (2) a recursive Bayesian estimation framework for estimating the parameters of a model flowfield using noisy flow measurements; and (3) a dynamic feedback control algorithm utilizing the estimated flowfield to steer the vehicle to a desired orientation with respect to the surrounding flow. The contributions are illustrated using numerical simulations in a uniform flowfield with unknown flowspeed and angle of attack; application of this estimation and control framework to non-uniform flows is ongoing.

Section II reviews the conformal mapping technique for modeling the flow around a streamlined body; it also reviews the empirical observability gramian formulation for measuring flowfield observability, presents a framework for analysis of sensor placement strategies using several optimality metrics of the observability gramian, and describes a recursive Bayesian filter framework for estimating flowfield parameters. Section III presents sensor-placement optimization results for a multi-sensor array, motivating the multisensor design by first analyzing the placement of a single 
sensor, and describes a feedback control algorithm to steer the body to a desired angle of attack. Section IV summarizes the results and ongoing work.

\section{BACKGROUND}

\section{A. Flow around a streamlined body}

This section uses techniques from potential flow theory and conformal mapping to describe a model of fluid flow past a streamlined body. The flowfield model is used to derive optimal sensor placement strategies for flowfield estimation and closed-loop control in Section III.

Consider a point $\xi \in \mathbb{C}$ in the complex plane. The coordinate transformation [11]

$$
z=\xi+\frac{b^{2}}{\xi} \in \mathbb{C},
$$

maps shapes according to the transformation variable $b \in \mathbb{R}$. For example [11], $\xi=R e^{i \theta}-\lambda$, where $\theta \in[0,2 \pi)$, defines a disk with radius $R$ offset along the real axis by $\lambda \in \mathbb{R}$. Choosing $b=R-\lambda$ maps the disk to a symmetric, streamlined body as shown in Figure 1. Note, the body shape is defined by the parameters $(R, \lambda)$ and the transformation (1).

The potential flow around a disk can be approximated using the sum of a uniform flow, a doublet, and a vortex [11],[14]. The corresponding velocity potential is [11],[14]

$$
w(\xi)=U \xi e^{-i \alpha}+\frac{R^{2}}{\xi+\lambda} U e^{i \alpha}-\frac{i \Gamma}{2 \pi} \ln (\xi+\lambda),
$$

where $U>0$ is the freestream speed of the uniform flow, $\alpha \in[0,2 \pi]$ is the angle of attack of the body, and $\Gamma \in \mathbb{R}$ is the circulation of the vortex. (Note the potential flow approximation is valid only for small angles of attack, so we take $\alpha \in\left[-15^{\circ}, 15^{\circ}\right]$ consistent with symmetric foils at low Reynolds numbers [15].) The conjugate flow $f^{*}=u-i v$ at $\xi$ is the gradient of the velocity potential (2) [11], i.e.,

$$
f^{*}(\xi)=\frac{\partial w}{\partial \xi}=U e^{-i \alpha}-\frac{R^{2}}{(\xi+\lambda)^{2}} U e^{i \alpha}-\frac{i \Gamma}{2 \pi(\xi+\lambda)} .
$$

Using (1) and (3), the conjugate flow around the body in $z$ coordinates is

$$
\begin{aligned}
f^{*}(z) & =\frac{\partial w}{\partial \xi}\left(\frac{\partial z}{\partial \xi}\right)^{-1} \\
& =\left(U e^{-i \alpha}-\frac{R^{2}}{(\xi+\lambda)^{2}} U e^{i \alpha}-\frac{i \Gamma}{2 \pi(\xi+\lambda)}\right)\left(1-\frac{b^{2}}{\xi^{2}}\right)^{-1} .
\end{aligned}
$$

The Kutta condition [11],[14] stipulates that the flow must be continuous around the body, which implies that the flow stagnates at the trailing edge of the body. This condition determines the vortex circulation $\Gamma$ by evaluating $f^{*}(z)=0$ in (4) at $\xi=R e^{i 0}-\lambda$ and solving for $\Gamma$ to obtain [11], [14]

$$
\Gamma=-4 \pi R U \sin \alpha \text {. }
$$

Note that (4) provides the flow around the streamlined body in $\xi$ coordinates. The mapping from $z$ to $\xi$ is dualvalued with one point inside the disk and one outside. We concern ourselves with the point outside the disk and solve (1) for $\xi$ using the quadratic equation to obtain [11]

$$
\xi(z)= \begin{cases}\frac{1}{2}\left(z+\sqrt{z^{2}-4 b^{2}}\right), & \text { if } \arg (z) \in(-\pi / 2, \pi / 2] \\ \frac{1}{2}\left(z-\sqrt{z^{2}-4 b^{2}}\right), & \text { if } \arg (z) \in(\pi / 2,3 \pi / 2] .\end{cases}
$$

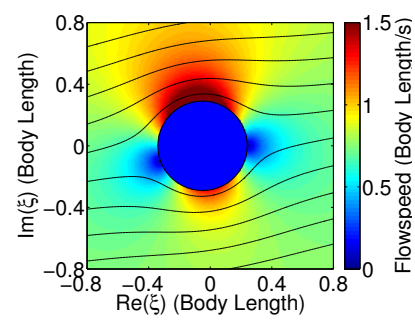

(a)

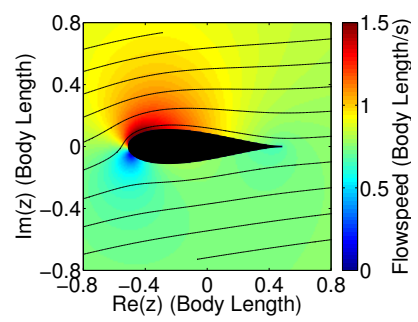

(b)
Fig. 1. (a) Flowfield around (a) a disk and (b) a fish-like streamlined body with $R=2.9$ and $\lambda=0.5$.

For $z=x+i y$ and $\xi=\xi_{x}+i \xi_{y}$, we have

$$
\begin{aligned}
\xi_{x}= & \frac{x}{2} \pm \frac{1}{2}\left[\left(x^{2}-y^{2}-4 b^{2}\right)^{2}+4 x^{2} y^{2}\right]^{1 / 4} \\
& \cos \left(\frac{1}{2} \tan ^{-1}\left(\frac{2 x y}{x^{2}-y^{2}-4 b^{2}}\right)\right)
\end{aligned}
$$

and

$$
\begin{aligned}
\xi_{y}= & \frac{y}{2} \pm \frac{1}{2}\left[\left(x^{2}-y^{2}-4 b^{2}\right)^{2}+4 x^{2} y^{2}\right]^{1 / 4} \\
& \sin \left(\frac{1}{2} \tan ^{-1}\left(\frac{2 x y}{x^{2}-y^{2}-4 b^{2}}\right)\right) .
\end{aligned}
$$

Combining equations (4), (5), and (6) gives the conjugate flow $f^{*}$ in $z$ coordinates [11] (omitted for brevity). Figure 1 shows the streamlines and flowspeeds along the circular and streamlined bodies calculated from (3) and (4), respectively. Note, due to the constraint imposed by the Kutta condition the flow around the cylinder in Figure 1(a) is asymmetric.

For a known body shape $(R, \lambda)$, the (uniform) flowfield (4) is parameterized by the freestream velocity $U$ and the angle of attack $\alpha$. We define the $M$-dimensional parameter vector ${ }^{1}$ $\boldsymbol{\Omega}=(U, \alpha)$, where $M=2$. Let $N$ be the number of sensor nodes in the array. By assimilating measurements collected at sensor locations $z_{i}, i=1, \ldots, N$, one can reconstruct the flowfield by estimating $\boldsymbol{\Omega}$. Performance of an estimation scheme is improved by optimizing the sensor locations for observing the flow. The following subsection describes a framework for evaluating candidate sensor-placement strategies using the empirical observability gramian.

\section{B. Observability Measures}

In linear systems theory, the singular values of the observability gramian determine the relative difficulty of determining the initial states of a linear system from the outputs generated over a time interval [16, p. 125-126]. Large singular values imply that it is not difficult to invert the mapping from outputs to initial states [6]. The unobservability index $v \triangleq 1 / \sigma_{\min }$ (the reciprocal of the smallest singular value of the observability gramian) provides a (scalar) measure of the relative ease in which an estimation scheme can determine the initial state of a system [6]. In stochastic estimation, a large value of $v$ implies that measurement noise will significantly impact the estimate error. Conversely, a small value of $v$ implies that the estimation error is less susceptible to measurement noise [6]. This measure of the observability

\footnotetext{
${ }^{1}$ We use bold fonts to represent a column matrix, e.g., of sensor positions $\mathbf{z}=\left[\begin{array}{llll}z_{1} & z_{2} & \ldots & z_{N}\end{array}\right]^{T}$, or a set of parameters, e.g., $\boldsymbol{\Omega}=\left(\Omega_{1}, \Omega_{2}, \ldots, \Omega_{M}\right)$.
} 
gramian is thus a suitable metric for comparing candidate sensor placements.

Several other measures of the observability gramian have been proposed as a metric for the sensor-placement problem [7],[13], including the trace, the determinant, and the trace of the inverse. Maximizing the trace corresponds to maximizing the $\mathscr{L}_{2}$ norm of the outputs [13], whereas maximizing the determinant corresponds to a maximization of independence between outputs [13]. Minimizing the trace of the inverse is comparable to minimizing the error covariance [13]. We compare these strategies by optimizing the diagonal elements of the observability gramian, which represent the observability of individual flowfield parameters.

Using the classical observability gramian on a nonlinear system requires linearization about an equilibrium solution. However, linearization may not adequately capture the inputoutput behavior of the nonlinear system over a desired operating region. Moreover, nonlinear observability analysis [17] can be complicated to perform. One alternative for determining the observability of a nonlinear system is to use the empirical observability gramian [6], also known as the observability covariance matrix [18],[19]. The empirical observability gramian maps the input-to-state and state-tooutput behavior of a nonlinear system more accurately than the observability gramian found by linearization [19] and, for systems depending on time-invariant parameters, the empirical observability gramian is closely related to the Fisher information matrix, [13] as shown below.

Let the vector $\mathbf{z}=\left[z_{1}, z_{1}, \ldots, z_{N}\right]^{T}$ denote the positions of all $N$ sensors. We assume that sensor $k$ collects independent measurements of the flowfield such that the output is

$$
\beta_{k}=f_{k}=u_{k}+i v_{k} \in \mathbb{C},
$$

where $u_{k}$ and $v_{k}$ are given by (4) evaluated at $z_{k}=x_{k}+i y_{k}$. The output from all $N$ sensors is

$$
\boldsymbol{\beta}=\left[\beta_{1}, \beta_{2}, \ldots, \beta_{N}\right]^{T} \in \mathbb{C}^{N} .
$$

Let $\varepsilon_{i}$ be a small perturbation of the $i^{\text {th }}$ parameter along the unit vector $\mathbf{e}_{i} \in \mathbb{R}^{M}$ and let $\boldsymbol{\Omega}_{0} \in \mathbb{R}^{M}$ be the nominal parameter values (i.e., the current best estimate). For a set of time-invariant parameters, the $(i, j)^{\text {th }}$ component of the $M \times M$ empirical observability gramian $W_{O}$ is ${ }^{2}$ [6]

$$
\begin{aligned}
W_{O}(i, j ; \mathbf{z})= & \frac{1}{4 \varepsilon_{i} \varepsilon_{j}}\left\langle\boldsymbol{\beta}^{+i}-\boldsymbol{\beta}^{-i}, \boldsymbol{\beta}^{+j}-\boldsymbol{\beta}^{-j}\right\rangle, \\
& i=1, \ldots, M, j=1, \ldots, M,
\end{aligned}
$$

where $\boldsymbol{\Omega}^{ \pm i}=\boldsymbol{\Omega}_{0} \pm \boldsymbol{\varepsilon}_{i} \mathbf{e}_{i}$ produces the output $\boldsymbol{\beta}^{ \pm i}=\boldsymbol{\beta}\left(\mathbf{z} ; \boldsymbol{\Omega}^{ \pm i}\right)$. Measures of the observability of a nonlinear system can be obtained by applying metrics such as the unobservability index to $W_{O}$. Note, to compute $W_{O}(i, j)$ for time-varying parameters $\boldsymbol{\Omega}=\boldsymbol{\Omega}(t)$ or for measurements collected over time, (11) is integrated over the duration of the sampling

\footnotetext{
${ }^{2}$ Let $\langle x, y\rangle \triangleq \operatorname{Re}\left(x^{*} y\right)$, where $x^{*}$ is the complex conjugate of $x$, denote the inner product of complex numbers $x$ and $y$. For complex vectors and matrices, the notation $\mathbf{X}^{*}$ represents the conjugate transpose of $\mathbf{X}$. The notation $G(i, j ; \boldsymbol{\alpha})$ represents the $(i, j)^{\text {th }}$ element of the matrix $G$, which is a function of the state $\boldsymbol{\alpha}$. The notation $g(\boldsymbol{\alpha} ; \boldsymbol{\Omega})$ represents a function $g(\cdot)$ that depends on the state variables $\boldsymbol{\alpha}$ and the parameters $\boldsymbol{\Omega}$.
}

trajectories. In this setting, although angle of attack $\alpha$ is ultimately controlled and therefore time-varying, we assume that it is static for the purposes of calculating observability and sensor placement.

For systems in which the output $\boldsymbol{\beta}(\mathbf{z} ; \boldsymbol{\Omega})$ is a continuous function of the parameters $\boldsymbol{\Omega}$, the observability gramian and the Fisher information matrix are closely related [13]. In the limit that $\varepsilon_{i} \rightarrow 0$, we have

$$
\lim _{\varepsilon_{i} \rightarrow 0} \frac{\boldsymbol{\beta}^{+i}-\boldsymbol{\beta}^{-i}}{2 \varepsilon_{i}}=\frac{\partial \boldsymbol{\beta}}{\partial \Omega_{i}} .
$$

For infinitesimally small perturbations, the empirical observability gramian $W_{O}$ in (11) is approximated by

$$
W_{O} \approx\left[\begin{array}{ccc}
\left\langle\frac{\partial \boldsymbol{\beta}}{\partial \Omega_{1}}, \frac{\partial \boldsymbol{\beta}}{\partial \Omega_{1}}\right\rangle & \ldots & \left\langle\frac{\partial \boldsymbol{\beta}}{\partial \Omega_{1}}, \frac{\partial \boldsymbol{\beta}}{\partial \Omega_{M}}\right\rangle \\
\vdots & \ddots & \vdots \\
\left\langle\frac{\partial \boldsymbol{\beta}}{\partial \Omega_{M}}, \frac{\partial \boldsymbol{\beta}}{\partial \Omega_{1}}\right\rangle & \ldots & \left\langle\frac{\partial \boldsymbol{\beta}}{\partial \Omega_{M}}, \frac{\partial \boldsymbol{\beta}}{\partial \Omega_{M}}\right\rangle
\end{array}\right] \in \mathbb{R}^{M \times M} .
$$

Let

$$
\mathbf{X} \triangleq\left[\frac{\partial \boldsymbol{\beta}}{\partial \Omega_{1}}, \ldots, \frac{\partial \boldsymbol{\beta}}{\partial \Omega_{M}}\right]^{T} \in \mathbb{C}^{M \times N} .
$$

Assuming the sensors are identical and independent, the sensor noise covariance matrix $\mathbf{R}$ is diagonal and given by $\mathbf{R}=R_{0} \mathbf{I}_{N \times N}$, where $R_{0}$ is the sensor noise variance. Under this assumption, the observability gramian (13) becomes

$$
W_{O} \approx \mathbf{X X}^{*}=R_{0} \mathbf{X R}^{-1} \mathbf{X}^{*} \triangleq R_{0} F,
$$

where $F$ is the Fisher information matrix [13]. $F$ provides a measure of the information content within a set of noisy measurements and is associated with the inverse of the covariance matrix [20].

\section{Bayesian Estimation of a Parameterized Flowfield}

This section presents a nonlinear estimation scheme to enable a sensor array to estimate unknown parameters of a model flowfield, such as the flowfield presented in Section IIA. Estimation of a spatiotemporal flowfield $f$ of the form (4) using noisy measurements of the flow can be accomplished by assimilating the measurements using a recursive Bayesian filter. For linear systems with Gaussian noise the optimal Bayesian filter is the Kalman filter, whereas for nonlinear systems with nonlinear noise models a common Bayesian filter is a particle filter [21]. In either case, the flow estimate is encapsulated in a state vector, which for example may contain the flow velocity $f=u+i v$ at each one of $P$ grid points. An alternative we pursue here is a state vector $\boldsymbol{\Omega}$ that contains only a set of $M \ll P$ parameters, from which the flowfield $f$ can be reconstructed. For example, the model (4) is defined by the parameters $\boldsymbol{\Omega}=(U, \alpha)$, such that the flowfield at $z_{k}$ is $f_{k}=u\left(z_{k} ; \boldsymbol{\Omega}\right)+i v\left(z_{k} ; \boldsymbol{\Omega}\right)$. For flowfields with a low-dimensional parameter space, the recursive Bayesian filter is advantageous because of its simplicity and ability to account for nonlinearities in both the flowfield model and measurement noise. This representation provides a significant reduction in computations, making it attractive for use in a dynamic feedback controller. (Note this representation is only possible for a parameterized flowfield.) 
The Bayesian formalism proceeds as follows [21]. Let $\hat{\mathbf{\Omega}}$ denote the parameter estimates and $\tilde{\beta}_{k}=u_{k}+\eta_{u, k}+i\left(v_{k}+\right.$ $\left.\eta_{v, k}\right) \in \mathbb{C}$ denote a noisy observation collected from the $k^{\text {th }}$ sensor at position $z_{k}$, where the noise $\eta_{i, k} \sim \mathscr{N}\left(0, \sigma_{i}^{2}\right)$ is normally distributed with zero mean and variance $\sigma_{i}^{2}$ for $i=$ $u, v$. Denote $\mathbf{A}=\left\{\tilde{\beta}_{1}, \ldots, \tilde{\beta}_{N}\right\}$ as the set of observations from all sensors. The posterior probability of the state $\boldsymbol{\Omega}$ given $\mathbf{A}$ is $p(\boldsymbol{\Omega} \mid \mathbf{A})=\kappa p(\boldsymbol{A} \mid \boldsymbol{\Omega}) p\left(\boldsymbol{\Omega} \mid \mathbf{A}_{0}\right)$, where $\kappa$ ensures that $p(\boldsymbol{\Omega} \mid \mathbf{A})$ has unit integral over the state space. The likelihood function $p(\mathbf{A} \mid \boldsymbol{\Omega})$ represents the conditional probability of the observations $\mathbf{A}$ given the state $\boldsymbol{\Omega}$ and $p\left(\boldsymbol{\Omega} \mid \mathbf{A}_{0}\right)$ represents the prior probability distribution. Note that, in the absence of observations or any information other than parameter bounds, the prior probability $p\left(\boldsymbol{\Omega} \mid \mathbf{A}_{0}\right)$ is uniform.

For each point $\boldsymbol{\Omega}$ in the $M$-dimensional state space, we choose a multivariate gaussian likelihood function, i.e.,

$$
\begin{aligned}
p\left(\tilde{\beta}_{k} \mid \mathbf{\Omega}\right)= & \frac{1}{2 \pi|\Sigma|^{1 / 2}} \exp \left[-\frac{1}{2}\left(f_{k}\left(z_{k} ; \boldsymbol{\Omega}\right)-\tilde{\beta}_{k}\right)^{*}\right. \\
& \left.\Sigma^{-1}\left(f_{k}\left(z_{k} ; \boldsymbol{\Omega}\right)-\tilde{\beta}_{k}\right)\right]
\end{aligned}
$$

where $\Sigma=\operatorname{diag}\left(\sigma_{u}^{2}, \sigma_{v}^{2}\right)$. Assuming measurements are taken from $N$ sensors, the posterior probability density of the state estimate $\boldsymbol{\Omega}$ is obtained using the joint measurement likelihood as follows:

$$
p(\mathbf{\Omega} \mid \mathbf{A})=\kappa\left(\prod_{k=1}^{N} p\left(\tilde{\beta}_{k} \mid \mathbf{\Omega}\right)\right) p\left(\boldsymbol{\Omega} \mid \mathbf{A}_{0}\right),
$$

where $p\left(\tilde{\beta}_{k} \mid \mathbf{\Omega}\right)$ is given by (16). The point $\hat{\mathbf{\Omega}}$ in parameter space corresponding to the maximum of the posterior probability $p(\mathbf{\Omega} \mid \mathbf{A})$ (i.e., the mode) provides the maximum likelihood estimate of the flowfield parameters.

Equation (17) represents spatial integration over the sensor array. Temporal integration is accomplished in the Bayesian framework by using the posterior at time step $t-\Delta t$ to generate the prior at time $t$, i.e.

$$
\begin{aligned}
p(\boldsymbol{\Omega}(t) \mid \overline{\mathbf{A}}(t))= & \kappa\left(\prod_{k=1}^{N} p\left(\tilde{\beta}_{k}(t) \mid \boldsymbol{\Omega}(t)\right)\right) \int p(\boldsymbol{\Omega}(t) \mid \boldsymbol{\Omega}(t-\Delta t)) \\
& p(\boldsymbol{\Omega}(t-\Delta t) \mid \overline{\mathbf{A}}(t-\Delta t)) \mathrm{d} \boldsymbol{\Omega}(t-\Delta t),
\end{aligned}
$$

where $\overline{\mathbf{A}}(t)=\{\mathbf{A}(t), \mathbf{A}(t-\Delta t), \ldots, \mathbf{A}(0)\}$ and the motion model $p(\boldsymbol{\Omega}(t) \mid \boldsymbol{\Omega}(t-\Delta t))$ updates the probability density function from $t-\Delta t$ to $t$ [22, p.372-375]. We define the motion matrix $\Psi$ and let $p(\boldsymbol{\Omega}(t) \mid \boldsymbol{\Omega}(t-\Delta t))=\mathscr{N}\left(\Psi \boldsymbol{\Omega}(t-\Delta t) ; \Sigma_{p}\right)$, where $\mathscr{N}\left(\Psi \boldsymbol{\Omega}(t-\Delta t) ; \Sigma_{p}\right)$ is normally distributed white noise with mean $\Psi \boldsymbol{\Omega}(t-\Delta t)$ and variance $\Sigma_{p}$.

\section{DYNAMIC FEEDBACK CONTROL WITH OPTIMAL SENSOR PlaCEMENTS}

This section presents results on sensor placement optimization, flowfield estimation, and control of the vehicle orientation relative to the flow. Section III-A calculates the optimal placement of a single sensor based on the optimality metrics presented in Section II-B. Section III-B suggests a sensor-placement strategy for an $\mathrm{N}$-sensor configuration based on the observation that the multi-sensor empirical observability gramian is the sum of the observability gramians produced by each sensor and Section III-C incorporates estimates from the recursive Bayesian filter in a dynamic feedback control that regulates the vehicle's angle of attack.

\section{A. Placement Optimization for a Single Sensor}

A first step in solving the sensor placement problem for a uniform and steady flowfield parameterized by $\boldsymbol{\Omega}=(U, \alpha)$ is to consider a single flow sensor placed a fixed distance from the body. Optimization of the placement of a single sensor is motivated by noting that the inner product used to calculate $W_{O}(i, j)$ is a linear operator. Due to linearity, the empirical observability gramian for an $N$-sensor configuration is the sum of the empirical observability gramians produced by each sensor, i.e.,

$$
W_{O}(i, j ; \mathbf{z})=\sum_{k=1}^{N} W_{O}\left(i, j ; z_{k}\right),
$$

where $W_{O}$ is found from (11). Consequently, the results of single sensor optimization are directly applicable to the design of a multi-sensor configuration in Section III-B.

Envisioning hair sensors that protrude outside the boundary layer, we calculate the observability gramian from a single measurement at $z_{1}$ using (11), where $\boldsymbol{\beta}=\beta_{1}$. Due to the linear dependence of the flowfield model (4) on the parameter $U$ and the definition of the empirical observability gramian (11), the perturbation value $\varepsilon_{U}$ does not appear in the analytic form of (11) (omitted for brevity). The element $W_{O}(1,1)$ corresponds to the squared ratio of the flow magnitude and the freestream parameter $U$, which represents a perturbation in $U$ and is maximized at the location of maximum flowspeed. Likewise, $W_{O}(2,2)$ corresponds to a perturbation in the angle of attack. Maximizing these elements individually gives insight into sensor configurations that maximize the observability of each flowfield parameter.

We calculate the observability gramian (11) as a function of the sensor location and evaluate the observability metrics described in Section II-B for a given parameter set $\boldsymbol{\Omega}$, assuming the sensor protrudes out from the body by 0.02 body lengths (BL). Figure 2 (left) shows the log of each dimensionless scoring metric plotted versus the polar angle $\arg \left(z_{k}\right)$ of the sensor placement for $\boldsymbol{\Omega}=(0.6 \mathrm{BL} / \mathrm{s}, 10 \mathrm{deg})$. Figure 2 (top right) shows streamlines of the flow around a body parameterized by $R=2.9$ and $\lambda=0.5$. The possible sensor placement positions are shown by the black line around the body, whereas the optimal sensor placements are color coded with respect to the metrics in Figure 2 (left). Optimization of $W_{O}(1,1)$ (blue) places the sensor at the location of the maximum flowspeed. The extrema of the remaining optimality metrics including the angle of attack element $W_{O}(2,2)$, unobservability index, trace, determinant, and the trace of the inverse (red) lie on or near the tip of the body $\left(\arg \left(z_{k}\right)=180^{\circ}\right)$, indicating that sensors placed in this region of the body maximize the independence between the angle of attack $\alpha$ and flowspeed $U$ in the measurement model (9) while minimizing the error covariance [13]. 


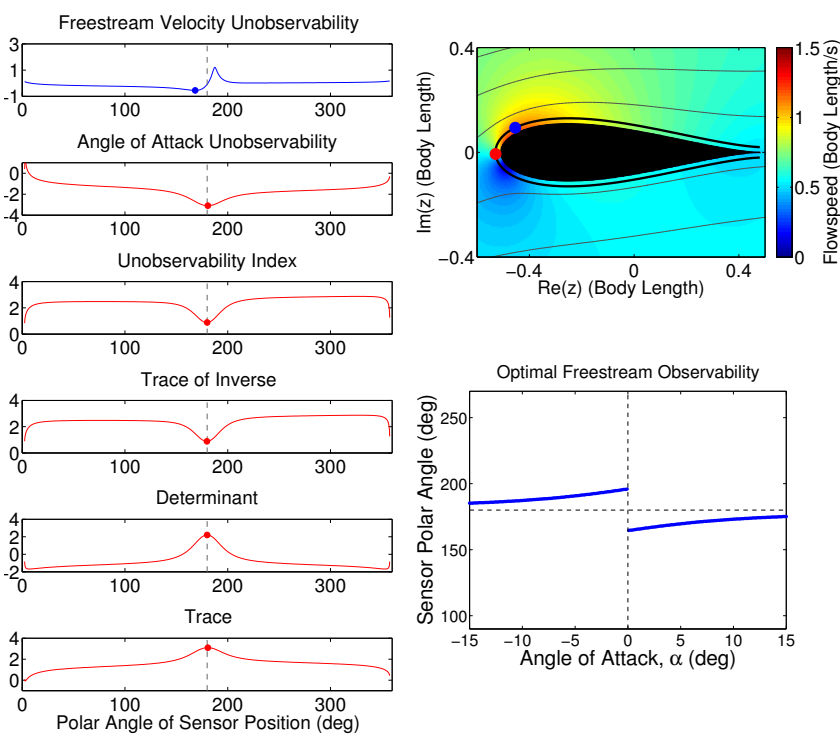

Fig. 2. Optimization results for one flow sensor: (left) log of optimality metrics versus the polar angle of the sensor placement near the fish body for $\boldsymbol{\Omega}=(0.6 \mathrm{BL} / \mathrm{s}, 10 \mathrm{deg})$; (top right) sensor placements of optimality metrics; (bottom right) optimal polar angle of free-stream observability sensor $U$ versus angle of attack.

Performing the observability analysis for flowspeeds $U \in$ $[0.01,1] \mathrm{BL} / \mathrm{s}$ (not shown) and angle of attack $\alpha \in$ $\left[-15^{\circ}, 15^{\circ}\right]$ indicates how sensor placement varies with $\boldsymbol{\Omega}$. Since the flow (4)-(5) is linearly dependent on $U$, the observability of the freestream parameter $U$ increases with $U$ at any $z_{k}$ or $\alpha$. Figure 2 (bottom right) shows the optimal polar angle of the sensor for varying angles of attack and fixed velocity parameter $U=0.6 \mathrm{BL} / \mathrm{s}$ when optimizing over the freestream velocity element of the observability gramian $W_{O}(1,1)$. Recall that $W_{O}(1,1)$ corresponds to the observability of the free stream velocity parameter $U$ and note that the optimal sensor placement varies with $\alpha$ to follow the point of maximum flowspeed near the body. The optimal placement with respect to the remaining metrics lies within $\pm 3^{\circ}$ of the tip of the body.

\section{B. Placement Optimization for Multiple Sensors}

This section considers the optimization of a multi-sensor configuration. We assume the flowfield parameters $\boldsymbol{\Omega}$ lie within a predefined range and each of the $N$ sensors are placed a fixed distance $0.02 \mathrm{BL}$ from the body. Under these assumptions the observability gramian from measurements at $\mathbf{z}=\left[z_{1}, \ldots, z_{N}\right]^{T}$ is given by (11), where $\boldsymbol{\beta}$ is given by (10), (9), and (4).

A desirable configuration optimizes observability over a range of the parameter space $\boldsymbol{\Omega}$, assuming the flowfield model used in this paper is valid for angles of attack $\alpha \in$ $\left[-15^{\circ}, 15^{\circ}\right]$. At any given $\alpha$ we desire a configuration in which at least one sensor is optimally placed. For this reason we choose a configuration (assuming $N$ is odd) in which $N-1$ sensors are placed symmetrically about the body with the polar angle of each sensor optimizing at angles of attack in $15^{\circ} / N$ intervals. A single sensor is placed at the tip of

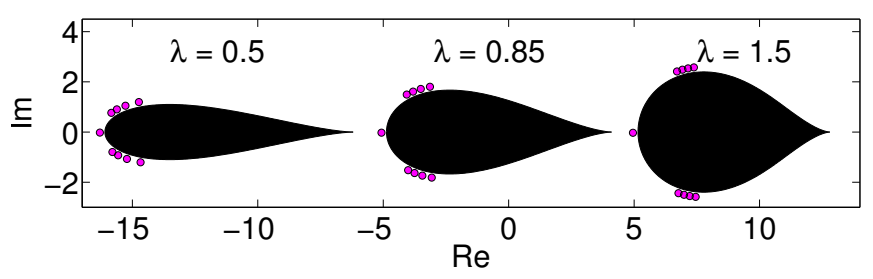

Fig. 3. Optimized sensor configurations for three fish-like body shapes parameterized by $R=2.9$ and $\lambda=0.5,0.85$, and 1.5 , respectively.

the body to optimize the remaining metrics. This sensor configuration therefore satisfies all of the optimality criterion along equal intervals of the flowfield parameter space $\boldsymbol{\Omega}$. Figure 3 shows the optimized sensor configuration scheme for $N=9$ sensors and three streamined bodies parameterized by $R=2.9$ and $\lambda=0.5,0.85$, and 1.5 , respectively.

\section{Dynamic Feedback Control of Angle of Attack}

By employing recursive Bayesian filtering with an optimized sensor placement configuration the flowfield can be estimated even in the presence of measurement noise. This section uses the estimated flowfield parameters from the recursive Bayesian filter in a dynamic feedback control that stabilizes the vehicle about zero angle of attack. Assuming the body has a control surface or motor (e.g., rudder, fin, or gantry system) that regulates the body's turn rate, the angle of attack $\alpha(t)$ is modeled kinematically by the equation $\dot{\alpha}=\gamma$, where the control $\gamma=\gamma(\hat{\boldsymbol{\Omega}})$ is designed using feedback of the parameter estimates $\hat{\boldsymbol{\Omega}}=(\hat{U}, \hat{\alpha})$. Suppose the estimated angle of attack can be modeled as $\hat{\alpha}=\alpha+w$, where the perturbation $w \leq \delta$ is bounded. Using a proportional control

$$
\gamma(t)=-K \hat{\alpha}, \quad K>0,
$$

gives the closed-loop equation

$$
\dot{\alpha}=-K(\alpha+w) .
$$

Use of the Lyapunov function $V=\alpha^{2} / 2$ reveals that if $w=0$ then $\alpha=0$ is exponentially stable (see, e.g. [23, p. 114]). For $w \neq 0, \alpha(t)$ is uniformly, ultimately bounded for $|w| \leq \delta$ with ultimate bound $|\alpha(t)| \leq \delta / K$ [23, p. 347].

Figure 4 shows a first-order, discrete-time simulation of the closed-loop control (20) using $N=9$ sensors at the optimal locations for $\alpha \in\left[-15^{\circ}, 15^{\circ}\right]$ and time step $\mathrm{d} t=$ 0.1 seconds. The estimated $\hat{\alpha}$ is provided by the recursive Bayesian filter (18) and the control gain is $K=1$. We assume the sensors collect noisy measurements of the flow with noise $\sigma_{u}=\sigma_{v}=0.25 \mathrm{BL} / \mathrm{s}$ and that the Bayesian filter is implemented with the motion matrix $\Psi=\Delta t \operatorname{diag}\left([0 \gamma]^{T}\right)$ and process noise $\Sigma_{p}=\operatorname{diag}\left(\left[0.25(\mathrm{BL} / \mathrm{s})^{2}, 0.03(\mathrm{rad} / \mathrm{s})^{2}\right]\right)$. Figures 4(a) and 4(b) show the initial and final orientation of the streamlined body, respectively; Figures 4(c) and 4(d) show the marginal probability densities ${ }^{3}$ of the angle of attack $\alpha$ and freestream velocity parameter $U$. The actual flowspeed

\footnotetext{
${ }^{3}$ The marginal probability density is achieved by summing a multidimensional probability density over a subset of dimensions.
} 


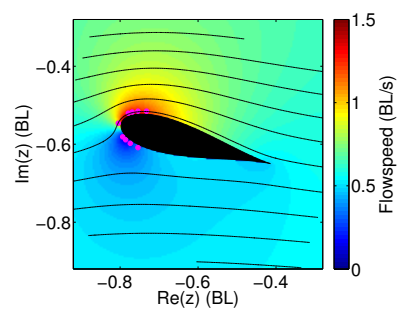

(a)

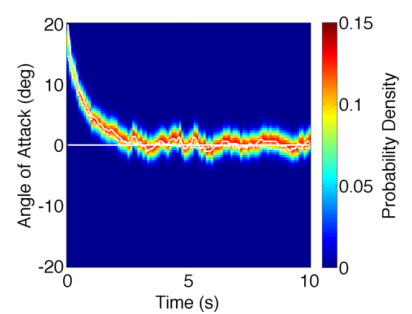

(c)

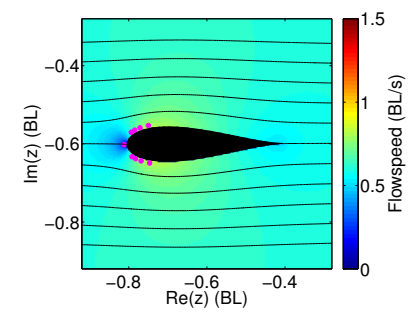

(b)

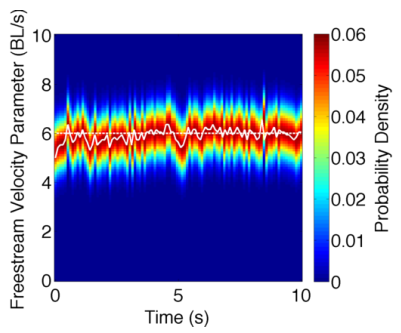

(d)
Fig. 4. Simulation of the dynamic control (20). (a,b) The initial and final orientation of the streamlined body; $(c, d)$ marginal probability densities of the recursive Bayesian filter.

$U=0.6 \mathrm{BL} / \mathrm{s}$ and angle of attack are depicted by the dashed white lines; the parameter estimates are represented by a solid white line. Sensor positions are denoted by magenta circles in Figures 4(a) and 4(b). Note that the control (20) orients the body toward $\alpha=0$ with estimation errors causing small deviations from zero angle of attack, consistent with the boundedness analysis above.

\section{CONCLUSION}

This paper derives observability-based sensor placement strategies for flowfield estimation and control of a streamlined vehicle in a uniform flowfield. Velocity potentials and conformal mapping model the flow around a streamlined body and measures of the empirical observability gramian optimize sensor configuration strategies for flowfield observability. A Bayesian filter framework estimates the parameterized flow assuming sensors collect noisy flow velocity measurements and the estimated flowfield parameters are used in a dynamic feedback controller to steer the vehicle to zero angle of attack.

Results suggest that the optimal location of a single sensor is the front of the vehicle. Of the six optimality metrics evaluated, five suggest placement within $\pm 3^{\circ}$ of the tip for $\alpha \in\left[-15^{\circ}, 15^{\circ}\right]$ and the final metric, which measures flowfield sensitivity to perturbations in the freestream velocity parameter, is maximized at the point of highest flow magnitude. To optimize a multi-sensor configuration, we incorporate the single sensor results and exploit the linearity of the empirical observability gramian to present a placement strategy in which one sensor is located at the nose of the body and the remaining sensors are distributed around the body optimally for varying angles of attack.

Potential flow models provide a first step for analytical derivation of sensor placement strategies, but represent a highly idealized model of real underwater flow environ- ments. Ongoing works seeks to validate the sensor placement strategies of this paper experimentally and with high fidelity computational fluid models. In addition, we seek to derive additional bio-inspired control algorithms to emulate fish behaviors such as station holding.

\section{ACKNOWLEDGMENTS}

The authors would like to acknowledge Sheryl Coombs, J. Sean Humbert, Xiaobo Tan, Amanda Chicoli, Frank Lagor, and Joshua Brulé for discussions related to this work.

\section{REFERENCES}

[1] X. Chen, G. Zhu, X. Yang, D. L. Hung, and X. Tan, "Modelbased estimation of flow characteristics using an ionic polymer-metal composite beam," IEEE/ASME Trans. on Mechatronics, vol. 18, no. 3, pp. 932-943, June 2013.

[2] C. Phelan, J. Tangorra, G. Lauder, and M. Hale, "A biorobotic model of the sunfish pectoral fin for investigations of fin sensorimotor control," Bioinspiration and Biomimetics, vol. 5, 2010.

[3] Z. Ren and K. Mohseni, "A model of the lateral line of fish for vortex sensing," Bioinspiration \& Biomimetics, vol. 7, no. 3, May 2012.

[4] T. Salumae and M. Kruusmaa, "Flow-relative control of an underwater robot," Proc. of the Royal Soc. A, vol. 469, no. 2153, March 2013.

[5] R. Venturelli and et. al., "Hydrodynamic pressure sensing with an artificial lateral line in steady and unsteady flows," Bioinspiration \& Biomimetics, vol. 7, no. 3, Apr. 2012.

[6] A. Krener and K. Ide, "Measures of unobservability," in Proc. 48th IEEE Conf. Decision and Cont., Shanghai, China, Dec. 2009, pp. 6401-6406.

[7] A. K. Singh and J. Hahn, "Determining optimal sensor locations for state and parameter estimation for stable nonlinear systems," Industrial and Eng. Chem. Research, vol. 44, no. 15, pp. 5645-5659, June 2005.

[8] H. Salman, L. Kuznetsov, C. Jones, and K. Ide, "A method of assimilating Lagrangian data into a shallow-water-equation ocean model," Monthly Weather Review, vol. 134, pp. 1081-1101, Apr. 2006.

[9] K. Willcox, "Unsteady flow sensing and estimation via the gappy proper orthogonal decomposition," Computers and Fluids, vol. 35, pp. 208-226, 2006.

[10] J. Olszewski, M. Haehnel, M. Taguchi, and J. C. Liao, "Zebrafish larvae exhibit rheotaxis and can escape a continuous suction source using their lateral line," PLOS ONE, vol. 7, no. 5, p. e36661, 052012.

[11] R. L. Panton, Incompressible Flow. John Wiley and Sons, 1984.

[12] A. Klein and H. Bleckmann, "Determination of object position, vortex shedding frequency and flow velocity using artificial lateral line canals," Beilstein J. Nanotechnology, vol. 2, pp. 276-283, June 2011.

[13] A. V. Wouwer, N. Point, S. Porteman, and M. Remy, "An approach to the selection of optimal sensor locations in distributed parameter systems," J. of Proc. Cont., vol. 10, no. 4, pp. 291-300, Aug. 2000.

[14] J. D. Anderson, Fundamentals of Aerodynamics, 5th ed. McGrawHill, Feb. 2011.

[15] L. Zhen, H. Beom-soo, K. Moo-rong, and J. Ji-yuan, "Experimental and numerical study for hydrodynamic characteristics of an oscillating hydrofoil," J. of Hydrodynamics, vol. 20, no. 3, pp. 280-287, 2008.

[16] S. Skogestad and I. Postlethwaite, Multivariable Feedback Control: Analysis and Design. West Sussex, England: Wiley and Sons, 1996.

[17] R. Hermann and A. J. Krener, "Nonlinear controllability and observability," IEEE Trans. Auto. Control, vol. 22, no. 5, pp. 728-740, 1977.

[18] S. Lall, J. Marsden, and S. Glavaski, "A subspace approach to balanced truncation for model reduction of nonlinear control systems," Int. J. of Robust and Nonlinear Cont., vol. 12, no. 6, pp. 519-535, 2002.

[19] J. Hahn and T. F. Edgar, "A gramian based approach to nonlinear quantification and model classification," Industrial and Engineering Chemistry Research, vol. 40, pp. 5724-5731, 2001.

[20] D. Ucinski, Optimal Measurement Methods for Distributed Parameter System Identification, 1st ed. CRC Press, Aug. 2004.

[21] N. Bergman, "Recursive Bayesian estimation navigation and tracking applications," Ph.D. dissertation, Department of Electrical Engineering, Linkoping University, Sweden, 1999.

[22] Y. Bar-Shalom, X. Rong Li, and T. Kirubarajan, Estimation with Applications to Tracking and Navigation: Theory Algorithms and Software, 1st ed. Wiley-Interscience, June 2001.

[23] H. K. Khalil, Nonlinear Systems, 3rd ed. Prentice Hall, 2002. 